\title{
RADIAL TOEPLITZ OPERATORS ON THE FOCK SPACE AND SQUARE-ROOT-SLOWLY OSCILLATING SEQUENCES
}

\author{
KEVIN ESMERAL ${ }^{1}$, EGOR A. MAXIMENKO $^{2}$
}

\begin{abstract}
In this paper we show that the $\mathrm{C}^{*}$-algebra generated by radial Toeplitz operators with $L_{\infty}$-symbols acting on the Fock space is isometrically isomorphic to the $\mathrm{C}^{*}$-algebra of bounded sequences uniformly continuous with respect to the squareroot-metric $\rho(j, k)=|\sqrt{j}-\sqrt{k}|$. More precisely, we prove that the sequences of eigenvalues of radial Toeplitz operators form a dense subset of the latter $\mathrm{C}^{*}$-algebra of sequences.
\end{abstract}

\section{INTRODUCTION}

Let $\mathcal{F}^{2}(\mathbb{C})$ be the Fock space (also known as the Segal-Bargmann space, see [2, 6, $12,24]$ ) consisting of all entire functions that are square integrable with respect to the Gaussian measure

$$
d \mathrm{~g}(z)=\frac{1}{\pi} e^{-|z|^{2}} d v(z), \quad z \in \mathbb{C},
$$

where $v$ is the usual Lebesgue measure on $\mathbb{C}$.

Given $\varphi \in L_{\infty}(\mathbb{C})$, the Toeplitz operator $T_{\varphi}$ with defining symbol $\varphi$ acts on the Fock space $\mathcal{F}^{2}(\mathbb{C})$ by the rule $T_{\varphi} f=\mathrm{P}(f \varphi)$, where $\mathrm{P}$ stays for the Bargmann projection from $L_{2}(\mathbb{C}, d \mathrm{~g})$ onto $\mathcal{F}^{2}(\mathbb{C})$. Operators of this kind have been extensively studied $[4,18$, $22,28]$, particularly in connection with quantum mechanics [7, 9], harmonic analysis $[1,10]$, etc.

The $\mathrm{C}^{*}$-algebra generated by Toeplitz operators with $L_{\infty}$-symbols is not commutative, however, there are classes of defining symbols that generate commutative $\mathrm{C}^{*}$ algebras of Toeplitz operators. In particular, many authors [3, 5, 16, 17, 18, 27] have studied the Toeplitz operators with radial defining symbols acting on various spaces of analytic or harmonic functions over the unit ball or over the whole space $\mathbb{C}^{n}$. The main reason is that these operators are diagonal in the monomial basis, which provides easy access to their properties.

For radial symbols on the unit disk $\mathbb{D}$, intensive investigation by Suárez [25] complemented by Grudsky, Maximenko and Vasilevski [17] showed that the $\mathrm{C}^{*}$-algebra generated by radial Toeplitz operators acting on the Bergman space $\mathcal{A}^{2}(\mathbb{D})$ is isometrically isomorphic to the $\mathrm{C}^{*}$-algebra $\mathrm{SO}\left(\mathbb{Z}_{+}\right)$consisting of the bounded sequences that

Date: August 12, 2021.

The work on the paper was partically supported by CONACyT and by IPN-SIP project 20150422.

2010 Mathematics Subject Classification. Primary 47B35; Secondary 30H20, 41A35.

Key words and phrases. Toeplitz operator, radial, Fock space, slowly oscillating sequence. 
slowly oscillate in the sense of R. Schmidt:

$$
\operatorname{SO}\left(\mathbb{Z}_{+}\right)=\left\{\sigma \in \ell_{\infty}\left(\mathbb{Z}_{+}\right): \lim _{\frac{j+1}{k+1} \rightarrow 1}\left|\sigma_{j}-\sigma_{k}\right|=0\right\},
$$

where $\mathbb{Z}_{+}=\mathbb{N} \cup\{0\}$. In other words, $\operatorname{SO}\left(\mathbb{Z}_{+}\right)$consists of bounded functions $\sigma: \mathbb{Z}_{+} \longrightarrow$ $\mathbb{C}$ uniformly continuous with respect to the logarithmic metric

$$
\eta(j, k)=|\ln (j+1)-\ln (k+1)| .
$$

This result extends to weighted Bergman spaces over the unit ball [3, 20]. Recent studies [11, 17, 19, 25] have given explicit descriptions of commutative $\mathrm{C}^{*}$-algebras generated by vertical and angular Toeplitz operators on Bergman spaces.

This paper focuses on studying the $\mathrm{C}^{*}$-algebra generated by radial Toeplitz operators acting on Fock spaces. It is well known [28] that the normalized monomials $e_{n}(z)=$ $z^{n} / \sqrt{n !}, n \in \mathbb{Z}_{+}$, form an orthonormal basis of $\mathcal{F}^{2}(\mathbb{C})$, and that the Toeplitz operators with bounded radial symbols are diagonal with respect to this basis [18]. Namely, if $a \in L_{\infty}\left(\mathbb{R}_{+}\right)$and $\varphi(z)=a(|z|)$ a.e. $z \in \mathbb{C}$, then $T_{\varphi} e_{n}=\gamma_{a}(n) e_{n}$, where

$$
\gamma_{a}(n)=\frac{1}{\sqrt{n !}} \int_{\mathbb{R}_{+}} a(\sqrt{r}) e^{-r} r^{n} d r, \quad n \in \mathbb{Z}_{+} .
$$

From this diagonalization we have that the $\mathrm{C}^{*}$-algebra $\mathcal{T}_{\text {rad }}$ generated by Toeplitz operators with radial $L_{\infty}$-symbols is isometrically isomorphic to the $\mathrm{C}^{*}$-algebra $\mathcal{G}$ generated by the set $\mathbb{5}$ of all sequences of the eigenvalues:

$$
\mathfrak{G}=\left\{\gamma_{a}: a \in L_{\infty}\left(\mathbb{R}_{+}\right)\right\} .
$$

The main result of this paper states that the uniform closure of 65 coincides with the $\mathrm{C}^{*}$-algebra $\operatorname{RO}\left(\mathbb{Z}_{+}\right)$consisting of all bounded sequences $\sigma: \mathbb{Z}_{+} \longrightarrow \mathbb{C}$ that are uniformly continuous with respect to the sqrt-metric

$$
\rho(m, n)=|\sqrt{m}-\sqrt{n}|, \quad m, n \in \mathbb{Z}_{+} .
$$

As a consequence, we obtain an explicit description of the $\mathrm{C}^{*}$-algebra $\mathcal{G}$ generated by (5):

$$
\mathcal{G}=\operatorname{RO}\left(\mathbb{Z}_{+}\right)
$$

Surprisingly for us, the $\mathrm{C}^{*}$-algebra $\mathcal{G}$ turns out to be wider than the class of sequences $\mathrm{SO}\left(\mathbb{Z}_{+}\right)$obtained for the radial case on weighted Bergman spaces.

The results of the paper can be generalized to radial Toeplitz operators on the multidimensional Fock space $\mathcal{F}^{2}\left(\mathbb{C}^{n},(\alpha / \pi)^{n} e^{-\alpha|z|^{2}} d v_{n}(z)\right)$; in this case the eigenvalue associated to the element $e_{\beta}$ of the canonical basis depends only on the length of the multiindex $\beta$, as in [17].

The paper is organized as follows. In Section 2 we have compiled some basic facts about radial Toeplitz operators in Fock space. In Sections 3 and 4 we introduce the class $\mathrm{RO}\left(\mathbb{Z}_{+}\right)$and prove that $\left(5\right.$ is contained in $\mathrm{RO}\left(\mathbb{Z}_{+}\right)$.

The major part of the paper is occupied by a proof that $\left(55\right.$ is dense in $\operatorname{RO}\left(\mathbb{Z}_{+}\right)$, see a scheme in Figure 1. Given a sequence $\sigma \in \operatorname{RO}\left(\mathbb{Z}_{+}\right)$, we extend it to a sqrt-oscillating function $f$ on $\mathbb{R}_{+}$(Proposition 3.4). After the change of variables $h(x)=f\left(x^{2}\right)$ we obtain a bounded and uniformly continuous function $h$ on $\mathbb{R}$. In Section 5, using Dirac sequences and Wiener's division lemma, we show that functions from $C_{b, u}(\mathbb{R})$ can be 
uniformly approximated by convolutions $k * b$, where $k$ is a fixed $L_{1}(\mathbb{R})$-function whose Fourier transform does not vanish on $\mathbb{R}$. This construction will be applied when $k$ is the heat kernel $H$. In Section 6 we examine the asymptotic behavior of the eigenvalues' sequences $\gamma_{a}$. It is shown there that after change of variables $\sqrt{n}=x$, the function $x \mapsto \gamma_{a}\left(x^{2}\right)$, for $x$ sufficiently large, is close to the convolution of the symbol $a$ with the heat kernel $H$. In Section 7 first we show that $c_{0}\left(\mathbb{Z}_{+}\right)$coincides with the uniform closure of the set $\left\{\gamma_{a}: a \in L_{\infty}\left(\mathbb{R}_{+}\right), \lim _{r \rightarrow \infty} a(r)=0\right\}$. After that, gathering together all the pieces, we obtain the main result. Finally, in Section 8 we describe a class of generating symbols bigger than $L_{\infty}(\mathbb{R})$, with eigenvalues' sequences still belonging to $\operatorname{RO}\left(\mathbb{Z}_{+}\right)$, and construct an unbounded generating symbol $a$ such that $\gamma_{a} \in \ell_{\infty}\left(\mathbb{Z}_{+}\right) \backslash \operatorname{RO}\left(\mathbb{Z}_{+}\right)$.

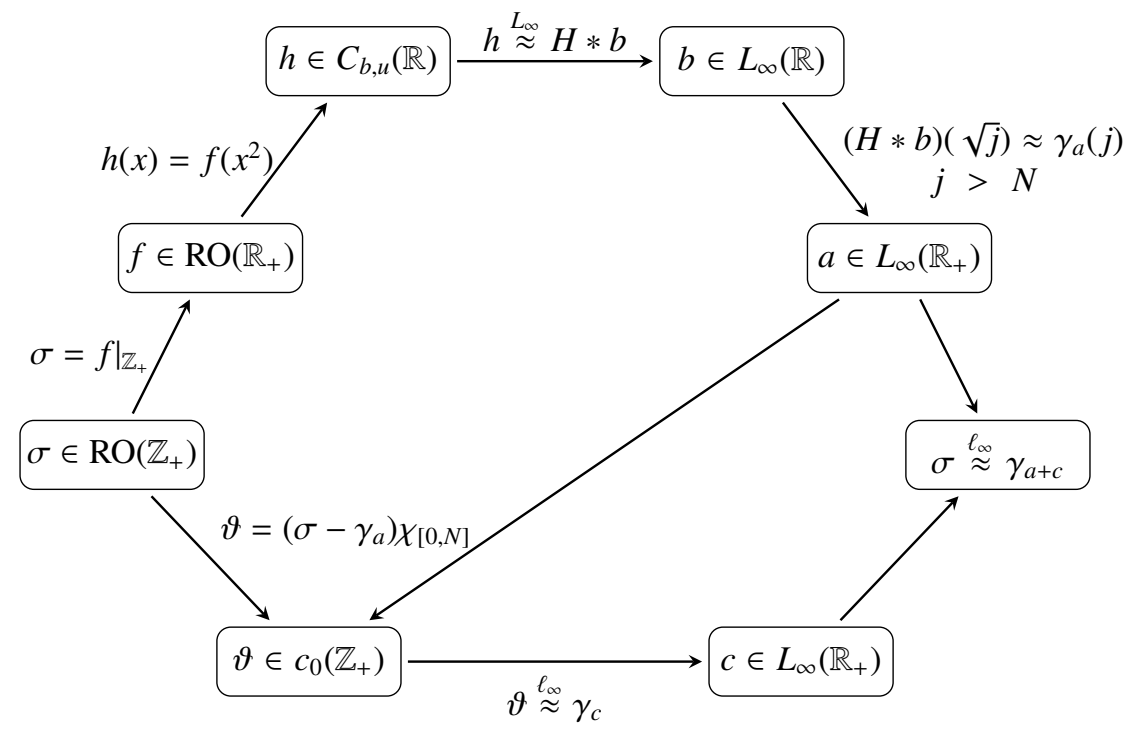

FIGURE 1. Scheme of the proof of density: the upper chain represents the approximation of $\sigma(j)$ for large values of $j(j>N)$, and the lower one corresponds to the uniform approximation of the sequence $\sigma-\gamma_{a}$ multiplied by the characteristic function $\chi_{[0, N]}$. 


\section{RADIAL TOEPLITZ OPERATORS}

In this section we compile some basic facts on Toeplitz operators with radial symbols from $L_{\infty}(\mathbb{C})$ acting on the Fock space $\mathcal{F}^{2}(\mathbb{C})$. Essentially, we repeat for the Fock space the facts stated by Zorboska [27] for the Bergman space over the unit disk, adding some ideas from [18].

Let $t \in \mathbb{R}$, and $U_{t}: \mathcal{F}^{2}(\mathbb{C}) \rightarrow \mathcal{F}^{2}(\mathbb{C})$ be the unitary operator given by the composition of functions with the rotation by the angle $t$ around the origin in the negative direction:

$$
\left(U_{t} f\right)(z)=f\left(e^{-i t} z\right), \quad z \in \mathbb{C} .
$$

For $S \in \mathcal{B}\left(\mathcal{F}^{2}(\mathbb{C})\right)$ we denote by $\operatorname{Rad}(S)$ the radialization of $S$ defined by

$$
\operatorname{Rad}(S)=\frac{1}{2 \pi} \int_{0}^{2 \pi} U_{-t} S U_{t} d t,
$$

where the integral is understood in the weak sense.

Definition 2.1 (radial operator acting on the Fock space). Let $S \in \mathcal{B}\left(\mathcal{F}^{2}(\mathbb{C})\right.$ ). The operator $S$ is said to be radial if it is invariant under rotations, that is, if for every $t \in[0,2 \pi)$,

$$
S U_{t}=U_{t} S \text {. }
$$

Observe that $S \in \mathcal{B}\left(\mathcal{F}^{2}(\mathbb{C})\right)$ is radial if and only if $\operatorname{Rad}(S)=S$.

Definition 2.2 (radial function). A function $\varphi \in L_{\infty}(\mathbb{C})$ is called radial if there exists $a \in L_{\infty}\left(\mathbb{R}_{+}\right)$such that $\varphi(z)=a(|z|)$ a.e. $z \in \mathbb{C}$.

Definition 2.3 (the radialization of a function). Let $\varphi \in L_{\infty}(\mathbb{C})$. The function $\operatorname{rad}(\varphi)$ given by

$$
\operatorname{rad}(\varphi)(z)=\frac{1}{2 \pi} \int_{0}^{2 \pi} \varphi\left(e^{i t} z\right) d t
$$

is called the radialization of $\varphi$.

By the periodicity of the mapping $t \mapsto e^{i t}$, the formula (2.4) can rewritten as

$$
\operatorname{rad}(\varphi)(z)=\frac{1}{2 \pi} \int_{0}^{2 \pi} \varphi\left(e^{i t}|z|\right) d t
$$

Lemma 2.4 (criterion for a function to be radial). A function $\varphi \in L_{\infty}(\mathbb{C})$ is radial if and only if $\varphi(z)=\operatorname{rad}(\varphi)(z)$ a.e. $z \in \mathbb{C}$.

Proof. Suppose that $\varphi \in L_{\infty}(\mathbb{C})$ is radial, i.e. there exists $a \in L_{\infty}\left(\mathbb{R}_{+}\right)$such that $\varphi(z)=a(|z|)$ a.e. $z \in \mathbb{C}$. Therefore, by (2.5) and by Fubini's theorem one gets that

$$
\begin{aligned}
\int_{\mathbb{C}} \operatorname{rad}(\varphi)(w) w^{n} \bar{w}^{m} d \mathrm{~g}(w) & =\frac{1}{2 \pi} \int_{0}^{2 \pi} \int_{\mathbb{R}_{+}} \int_{0}^{2 \pi} r^{n+m+1} e^{-r^{2}} \varphi\left(e^{i \alpha} r\right) e^{i \beta(n-m)} d \alpha d r d \beta \\
& =\frac{1}{2 \pi} \int_{0}^{2 \pi} \int_{\mathbb{R}_{+}} \int_{0}^{2 \pi} r^{n+m+1} e^{-r^{2}} a\left(\left|e^{i \alpha} r\right|\right) e^{i \beta(n-m)} d \alpha d r d \beta \\
& =\left(\int_{\mathbb{R}_{+}} a(r) r^{n+m+1} e^{-r^{2}} d r\right)\left(\int_{0}^{2 \pi} e^{i \beta(n-m)} d \beta\right) \\
& =\int_{\mathbb{C}} \varphi(w) w^{n} \bar{w}^{m} d \mathrm{~g}(w), \quad n, m \in \mathbb{Z}_{+} .
\end{aligned}
$$


Now, since $\operatorname{rad}(\varphi)-\varphi$ belongs to $L_{2}(\mathbb{C}, d \mathrm{~g})$ and the span of $\left\{w^{m} \bar{w}^{n}: m, n \in \mathbb{Z}_{+}\right\}$is dense in $L_{2}(\mathbb{C}, d g)$, we obtain that $\operatorname{rad}(\varphi)(z)=\varphi(z)$ a.e. $z \in \mathbb{C}$.

Conversely, if $\varphi(z)=\operatorname{rad}(\varphi)(z)$ a.e $z \in \mathbb{C}$, then by (2.5) one gets that $\varphi(z)=\operatorname{rad}(\varphi)(|z|)$ for a.e. $z \in \mathbb{C}$, which means that the condition of Definition 2.2 holds with $a(r)=$ $\operatorname{rad}(\varphi)(r)$.

The Berezin transform [6, 27] plays an important role in the description of properties of bounded operators, in particular for Toeplitz operators. The Berezin transform of a bounded operator $S$ on the Fock space $\mathcal{F}^{2}(\mathbb{C})$ is the function $\widetilde{S}$ defined by

$$
\widetilde{S}(z)=\frac{\left\langle S k_{z}, k_{z}\right\rangle}{\left\langle k_{z}, k_{z}\right\rangle}, \quad z \in \mathbb{C},
$$

where the function $k_{z}(w)=e^{\bar{z} w}$ is the reproducing kernel of $\mathcal{F}^{2}(\mathbb{C})$.

The next result provides a criterion for an operator to be radial. It mimics a result given by Zorboska [27] for operators on the Bergman space over the unit disk.

Theorem 2.5 (criterion of radial operators). Let $S \in \mathcal{B}\left(\mathcal{F}^{2}(\mathbb{C})\right)$. The following conditions are equivalent.

(i) $S$ is radial.

(ii) $S$ is a diagonal operator with respect to the monomial basis.

(iii) The Berezin transform $\widetilde{S}$ is a radial function.

An easy computation shows that $\operatorname{rad}(\widetilde{\varphi})=\widetilde{\operatorname{rad}(\varphi)}=\widetilde{T_{\operatorname{rad}(\varphi)}}$, for each $\varphi \in L_{\infty}(\mathbb{C})$. Thus, by Theorem 2.5 and by injectivity of Berezin transform the following criterion holds.

Proposition 2.6. Let $\varphi \in L_{\infty}(\mathbb{C})$. The Toeplitz operator $T_{\varphi}$ is radial if and only if $\varphi$ is a radial function.

\section{SQRT-oscillating SEQUences}

In this section we introduce formally the sets of sequences $\operatorname{RO}\left(\mathbb{Z}_{+}\right)$and functions $\operatorname{RO}([0,+\infty))$. We also show that the sequences of the class $\operatorname{RO}\left(\mathbb{Z}_{+}\right)$can be extended to functions of the class $\operatorname{RO}([0,+\infty))$.

Define $\rho: \mathbb{Z}_{+} \times \mathbb{Z}_{+} \rightarrow[0,+\infty)$ by

$$
\rho(m, n)=|\sqrt{m}-\sqrt{n}| .
$$

The function $\rho$ is a metric on $\mathbb{Z}_{+}$because it is obtained from the usual metric

$$
d: \mathbb{R}_{+} \times \mathbb{R}_{+} \rightarrow[0,+\infty), \quad d(t, u):=|t-u|,
$$

via the injective function $\mathbb{Z}_{+} \rightarrow \mathbb{R}_{+}, m \mapsto \sqrt{m}$.

The modulus of continuity with respect to the square-root-metric $\rho$ of a complex sequence $\sigma=\left(\sigma_{n}\right)_{n \in \mathbb{Z}_{+}}$is the function $\omega_{\rho, \sigma}:[0,+\infty) \rightarrow[0,+\infty]$ given by the rule

$$
\omega_{\rho, \sigma}(\delta)=\sup \left\{\left|\sigma_{n}-\sigma_{m}\right|: m, n \in \mathbb{Z}_{+}, \rho(m, n) \leq \delta\right\} .
$$

We denote by $\operatorname{RO}\left(\mathbb{Z}_{+}\right)$the set of the bounded sequences that are uniformly continuous with respect to the sqrt-metric:

$$
\operatorname{RO}\left(\mathbb{Z}_{+}\right)=\left\{\sigma \in \ell_{\infty}\left(\mathbb{Z}_{+}\right): \lim _{\delta \rightarrow 0} \omega_{\rho, \sigma}(\delta)=0\right\} .
$$


Proposition 3.1. $\mathrm{RO}\left(\mathbb{Z}_{+}\right)$is a closed $C^{*}$-subalgebra of $\ell_{\infty}\left(\mathbb{Z}_{+}\right)$.

Proof. The proof of this fact runs as in [17, Proposition 3.8].

The following simple criterion shows that the Lipschitz-continuity of sequences (with respect to the metric $\rho$ ) can be described in terms of the differences between the adjacent elements.

Proposition 3.2. A sequence $\sigma:: \mathbb{Z}_{+} \rightarrow \mathbb{C}$ is Lipschitz continuous with respect to $\rho$ if and only if

$$
\sup _{n \in \mathbb{Z}_{+}}(\sqrt{n+1}|\sigma(n+1)-\sigma(n)|)<+\infty .
$$

Proof. Suppose that $\sigma$ is Lipschitz continuous with respect to $\rho$, that is, there exists $M>0$ such that $|\sigma(m)-\sigma(n)| \leq M \rho(m, n)$ for each $m, n \in \mathbb{Z}_{+}$. Applying this inequality in the particular case $m=n+1$, one gets

$$
\sqrt{n+1}|\sigma(n+1)-\sigma(n)| \leq M(\sqrt{n+1}-\sqrt{n}) \sqrt{n+1}=\frac{M \sqrt{n+1}}{\sqrt{n+1}+\sqrt{n}} \leq M .
$$

Conversely, suppose that $\sup _{n}(\sqrt{n+1}|\sigma(n+1)-\sigma(n)|)=M<+\infty$. Hence, if $n>$ $m$, then we "join" $m$ with $n$ by the chain of the intermediate elements and estimate the differences of the adjacent elements using the hypothesis:

$$
\begin{aligned}
|\sigma(m)-\sigma(n)| & \leq \sum_{k=m}^{n-1}|\sigma(k+1)-\sigma(k)|=\sum_{k=m}^{n-1} \frac{\sqrt{k+1}+\sqrt{k}}{\sqrt{k+1}+\sqrt{k}}|\sigma(k+1)-\sigma(k)| \\
& \leq 2 \sum_{k=m}^{n-1} \frac{\sqrt{k+1}}{\sqrt{k+1}+\sqrt{k}}|\sigma(k+1)-\sigma(k)| \leq 2 M \sum_{k=m}^{n-1} \frac{1}{\sqrt{k+1}+\sqrt{k}} \\
& =2 M \sum_{k=m}^{n-1}(\sqrt{k+1}-\sqrt{k})=2 M(\sqrt{n}-\sqrt{m})=2 M \rho(n, m) .
\end{aligned}
$$

The same upper estimate can be drawn for $m \geq n$. Thus, $\sigma$ is Lipschitz continuous with respect to $\rho$.

The square root metric $\rho$ can be extended to the set $[0,+\infty)$ :

$$
\rho(x, y)=|\sqrt{x}-\sqrt{y}| \text {. }
$$

Given a function $f:[0,+\infty) \rightarrow \mathbb{C}$, its modulus of continuity with respect to $\rho$ is defined by

$$
\omega_{\rho, f}(\delta)=\sup \{|f(x)-f(y)|: x, y \in[0,+\infty), \rho(x, y) \leq \delta\} .
$$

We denote by $\operatorname{RO}([0,+\infty))$ the $\mathrm{C}^{*}$-algebra of all bounded and uniformly continuous functions on $[0,+\infty)$ with respect to the extended square root metric $\rho$ :

$$
\operatorname{RO}([0,+\infty))=\left\{f \in C_{b, u}([0,+\infty)): \lim _{\delta \rightarrow 0} \omega_{\rho, f}(\delta)=0\right\} .
$$

If $f$ is a function of the class $\operatorname{RO}([0,+\infty))$, then, obviously, its restriction to $\mathbb{Z}_{+}$is a sequence of the class $\mathrm{RO}\left(\mathbb{Z}_{+}\right)$. We are going to show that every sequence of the class $\mathrm{RO}\left(\mathbb{Z}_{+}\right)$can be obtained in this manner. Our extension of sequences to functions is just the piecewise-linear interpolation with respect to the parameter $\tau(x)=\sqrt{x}$. 
Lemma 3.3. Let $\sigma: \mathbb{Z}_{+} \rightarrow \mathbb{C}$. Define $f:[0,+\infty) \rightarrow \mathbb{C}$ by

$$
f(x)=\sigma(n)+\frac{\tau(x)-\tau(n)}{\tau(n+1)-\tau(n)}(\sigma(n+1)-\sigma(n)),
$$

where $n=\lfloor x\rfloor$ and $\tau(x)=\sqrt{x}$. Then $\left.f\right|_{\mathbb{Z}_{+}}=\sigma,\|f\|_{\infty}=\|\sigma\|_{\infty}$ and for every $\delta \in(0,1]$

$$
\omega_{\rho, f}(\delta) \leq 3 \max \left(\omega_{\rho, \sigma}(\sqrt{\delta}), \sqrt{\delta} \omega_{\rho, \sigma}(1)\right) .
$$

Proof. Note that $f(x)$ in (3.6) is a convex combination of $\sigma(n)$ and $\sigma(n+1)$ :

$$
f(x)=\frac{\tau(n+1)-\tau(x)}{\tau(n+1)-\tau(n)} \sigma(n)+\frac{\tau(x)-\tau(n)}{\tau(n+1)-\tau(n)} \sigma(n+1) .
$$

The first two assertions of the proposition are obvious. Let us prove (3.7). Fix $\delta \in(0,1]$ and suppose that $x, y \geq 0$ with $\rho(x, y) \leq \delta$.

Case I: $n \leq x \leq y \leq n+1$ for some $n \in \mathbb{Z}_{+}$. In this case

$$
|f(x)-f(y)|=\frac{\tau(y)-\tau(x)}{\tau(n+1)-\tau(n)}|\sigma(n+1)-\sigma(n)| \leq \frac{\rho(x, y) \omega_{\rho, \sigma}(\rho(n, n+1))}{\rho(n, n+1)} .
$$

If $\rho(n, n+1) \leq \sqrt{\delta}$, then

$$
|f(x)-f(y)| \leq \frac{\rho(x, y)}{\rho(n, n+1)} \omega_{\rho, \sigma}(\sqrt{\delta}) \leq \omega_{\rho, \sigma}(\sqrt{\delta}) .
$$

If $\rho(n, n+1) \geq \sqrt{\delta}$, then

$$
|f(x)-f(y)| \leq \frac{\delta}{\sqrt{\delta}} \omega_{\rho, \sigma}(1)=\sqrt{\delta} \omega_{\rho, \sigma}(1) .
$$

In both subcases,

$$
|f(x)-f(y)| \leq \max \left(\omega_{\rho, \sigma}(\sqrt{\delta}), \sqrt{\delta} \omega_{\rho, \sigma}(1)\right) .
$$

Case II: $\lfloor x\rfloor=n<m=\lfloor y\rfloor$. Then $\rho(n+1, m) \leq \rho(x, y) \leq \delta$, and

$$
|f(x)-f(y)| \leq|f(x)-f(n+1)|+|f(n+1)-f(m)|+|f(m)-f(y)| .
$$

Applying the inequality $\rho(n+1, m) \leq \rho(x, y) \leq \delta$ and the result of Case I, we obtain

$$
|f(x)-f(y)| \leq \omega_{\rho, \sigma}(\delta)+2 \max \left(\omega_{\rho, \sigma}(\sqrt{\delta}), \sqrt{\delta} \omega_{\rho, \sigma}(1)\right)
$$

In both cases, (3.7) holds.

Proposition 3.4. Let $\sigma \in \mathrm{RO}\left(\mathbb{Z}_{+}\right)$and $f:[0,+\infty) \rightarrow \mathbb{C}$ be the extension of $\sigma$ defined by (3.6). Then $f \in \mathrm{RO}([0,+\infty))$.

Proof. The assumption $\sigma \in \mathrm{RO}\left(\mathbb{Z}_{+}\right)$guarantees that the right-hand side of (3.7) tends to 0 as $\delta$ tends to 0 .

Note that Lemma 3.3 and Proposition 3.4 stay true for every metric $\rho$ of the form $\rho(x, y)=|\tau(x)-\tau(y)|$, where $\tau:[0,+\infty) \rightarrow[0,+\infty)$ is a strictly increasing function satisfying $\tau(n+1)-\tau(n) \leq 1$ for every $n \in \mathbb{Z}_{+}$. In particular, applying this construction with $\tau(n)=\ln (n+1)$ we obtain another proof of [20, Theorem 2.3] about the class $\mathrm{SO}\left(\mathbb{Z}_{+}\right)$; the proof in [20] is based on the usual piecewise-linear interpolation. 


\section{SQRT-OSCILlating PROPERTy OF THE EIGENVALUes' SEQUENCES}

In this section we show that $\gamma_{a} \in \operatorname{RO}\left(\mathbb{Z}_{+}\right)$for all $a \in L_{\infty}\left(\mathbb{R}_{+}\right)$. From now on, we write the eigenvalues' sequence $\gamma_{a}$ as follows:

$$
\gamma_{a}(n)=\int_{\mathbb{R}_{+}} a(\sqrt{r}) K(n, r) d r, \quad \text { where } \quad K(n, r)=\frac{r^{n} e^{-r}}{n !}, \quad n \in \mathbb{Z}_{+} .
$$

The following proposition introduces a metric on $\mathbb{Z}_{+}$which is, in a certain sense, the most "natural" for the functions $\gamma_{a}$.

Proposition 4.1. Let $\kappa: \mathbb{Z}_{+} \times \mathbb{Z}_{+} \rightarrow[0,+\infty)$ be the function given by

$$
\kappa(m, n)=\sup _{\substack{a \in L_{\infty}\left(\mathbb{R}_{+}\right) \\\|a\|_{\infty}=1}}\left|\gamma_{a}(m)-\gamma_{a}(n)\right| .
$$

Then

$$
\kappa(m, n)=\int_{\mathbb{R}_{+}}|K(m, r)-K(n, r)| d r .
$$

Proof. For every $a \in L_{\infty}\left(\mathbb{R}_{+}\right)$and $m, n \in \mathbb{Z}_{+}$we have

$$
\left|\gamma_{a}(m)-\gamma_{a}(n)\right| \leq\|a\|_{\infty} \int_{\mathbb{R}_{+}}|K(m, r)-K(n, r)| d r .
$$

On the other hand, if $m$ and $n$ are fixed and $m \neq n$, we define $a_{0}: \mathbb{R}_{+} \rightarrow \mathbb{R}$ by $a_{0}(r)=$ $\operatorname{sign}(K(m, r)-K(n, r))$, thus $a_{0} \in L_{\infty}\left(\mathbb{R}_{+}\right)$with $\left\|a_{0}\right\|_{\infty}=1$, and

$$
\kappa(x, y) \geq\left|\gamma_{a_{0}}(m)-\gamma_{a_{0}}(n)\right|=\int_{\mathbb{R}_{+}}|K(m, r)-K(n, r)| d r .
$$

Lemma 4.2. For every $n \in \mathbb{N}$ we get

$$
\kappa(n-1, n)=\frac{2 n^{n} e^{-n}}{n !}
$$

Proof. Given $n \in \mathbb{N}$, we write $\kappa(n-1, n)$ using (4.3):

$$
\kappa(n-1, n)=\int_{0}^{+\infty}\left|\frac{r^{n-1} e^{-r}}{(n-1) !}-\frac{r^{n} e^{-r}}{n !}\right| d r=\int_{0}^{+\infty} \frac{r^{n-1} e^{-r}}{(n-1) !}\left|1-\frac{r}{n}\right| d r .
$$

Now the integral falls naturally into two parts:

$$
\begin{aligned}
\kappa(n-1, n) & =\frac{1}{(n-1) !}\left[\int_{0}^{+\infty} e^{-r}\left(\frac{r^{n}}{n}-r^{n-1}\right) d r+2 \int_{0}^{n} e^{-r}\left(r^{n-1}-\frac{r^{n}}{n}\right) d r\right] \\
& =\frac{2}{(n-1) !} \int_{0}^{n} e^{-r}\left(r^{n-1}-\frac{r^{n}}{n}\right) d r \\
& =\frac{2}{(n-1) !}\left[\int_{0}^{n} e^{-r} r^{n-1} d r-\int_{0}^{n} e^{-r} \frac{r^{n}}{n} d r\right] .
\end{aligned}
$$

Integrating by parts in the latter integral one gets (4.4).

Lemma 4.3. For each $n \in \mathbb{N}$ we have

$$
\kappa(n-1, n) \leq \sqrt{\frac{2}{\pi n}}
$$


Moreover,

$$
\lim _{n \rightarrow \infty}(\kappa(n-1, n) \sqrt{n})=\sqrt{\frac{2}{\pi}} .
$$

Proof. The upper bound (4.5) follows from the left part of the well-known estimates

$$
n^{n} e^{-n} \sqrt{2 n \pi} \leq n ! \leq n^{n} e^{-n} \sqrt{2 n \pi} e^{\frac{1}{12 n}} .
$$

The limit relation (4.6) is a consequence of Stirling formula.

Proposition 4.4. $\left(\mathfrak{5} \subseteq \mathrm{RO}\left(\mathbb{Z}_{+}\right)\right.$.

Proof. Let $a \in L_{\infty}\left(\mathbb{R}_{+}\right)$. Then for every $n \in \mathbb{Z}_{+}$

$$
\left|\gamma_{a}(n)\right| \leq\|a\|_{\infty} \int_{\mathbb{R}_{+}} K(n, r) d r=\|a\|_{\infty} .
$$

Furthermore, by definition (4.2) of $\kappa$ and Lemma 4.3, for every $n \in \mathbb{N}$

$$
\left|\sqrt{n}\left(\gamma_{a}(n)-\gamma_{a}(n-1)\right)\right| \leq\|a\|_{\infty} \kappa(n, n-1) \sqrt{n} \leq \sqrt{\frac{2}{\pi}}\|a\|_{\infty} .
$$

Thus $\gamma_{a}$ is Lipschitz continuous with respect to $\rho$ by Proposition 3.2.

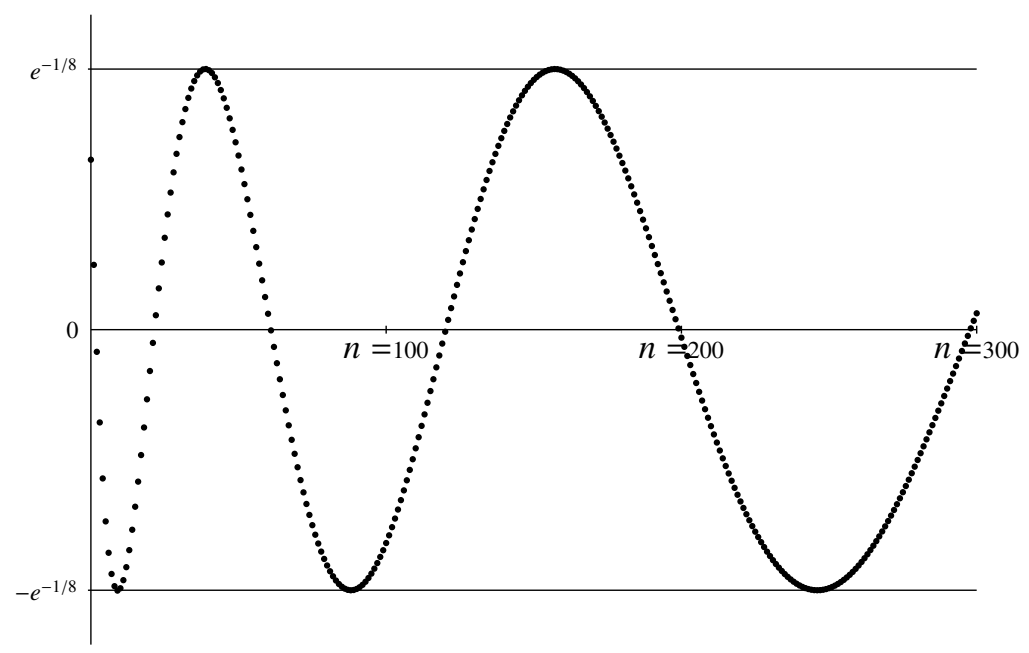

Figure 2. The first 301 values of the sequence $\gamma_{a}$ from Example 4.5.

Example 4.5 (sqrt-oscillating eigenvalues' sequence). Consider the Toeplitz operator generated by the radial symbol $a(r)=\cos r$. The corresponding eigenvalues are

$$
\gamma_{a}(n)=M(1+n, 1 / 2,-1 / 4),
$$

where $M={ }_{1} F_{1}$ is the Kummer's confluent hypergeometric function. Using Proposition 6.5 one can deduce an asymptotic formula for $\gamma_{a}(n)$, as $n \rightarrow \infty$ :

$$
\gamma_{a}(n)=e^{-1 / 8} \cos \sqrt{n}+o(1) \text {. }
$$

Figure 2 shows a plot of $\gamma_{a}(n)$ for $n=0,1, \ldots, 300$. 


\section{ApProximation OF UNIFORMLY CONTINUOUS FUNCTIONS BY CONVOLUTIONS}

In this section we recall a technique that permits to approximate bounded uniformly continuous functions by convolutions with a fixed kernel satisfying Wiener's condition. We could not find Proposition 5.4 in the literature, but it is based on well-known ideas and can be considered as a variation of the Wiener's Tauberian theorem. The constructions of this section can be generalized to abelian locally compact groups.

Definition 5.1 (Dirac sequences). A sequence $\left(h_{n}\right)_{n \in \mathbb{N}}$ of functions belonging to $L_{1}(\mathbb{R})$ is called a Dirac sequence if it satisfies the following conditions:

(a) For each $n \in \mathbb{N}$ and $x \in \mathbb{R}$, one gets $h_{n}(x) \geq 0$.

(b) For each $n \in \mathbb{N}$,

$$
\int_{\mathbb{R}} h_{n}(t) d t=1
$$

(c) For every $\delta>0$,

$$
\lim _{n \rightarrow+\infty} \int_{|x|>\delta} h_{n}(t) d t=0 .
$$

Example 5.2. The sequence $\left(h_{n}\right)_{n \in \mathbb{N}}$ given by

$$
h_{n}(x)=\frac{2 \sin ^{2}(n x)}{\pi n x^{2}}, \quad x \in \mathbb{R},
$$

is known as the Fejér's kernel on $\mathbb{R}$ and is a Dirac sequence. Moreover, it has a useful property that all the functions $\hat{h}_{n}$ have compact supports.

Since the Dirac sequences can be viewed as approximate identities, they provide a powerful tool to approximate functions. The next lemma is a well-known result for uniformly continuous functions, see for example [13, Proposition 2.42].

Lemma 5.3. Let $f \in C_{b, u}(\mathbb{R})$. If $\left(h_{n}\right)_{n \in \mathbb{N}}$ is a Dirac sequence, then

$$
\lim _{n \rightarrow \infty}\left\|f * h_{n}-f\right\|_{\infty}=0 .
$$

Proposition 5.4. Let $k \in L_{1}(\mathbb{R})$ satisfy Wiener's condition: $\hat{k}(t) \neq 0$ for each $t \in \mathbb{R}$. Then $\left\{k * f: f \in L_{\infty}(\mathbb{R})\right\}$ is a dense subset of $C_{b, u}(\mathbb{R})$.

Proof. It is known that $L_{1}(\mathbb{R}) * L_{\infty}(\mathbb{R})=C_{b, u}(\mathbb{R})$ (see [21, p. 283, 32-45]), hence every function in $\left\{k * f: f \in L_{\infty}(\mathbb{R})\right\}$ belongs to $C_{b, u}(\mathbb{R})$. Next, the density is proved by means of Wiener's Division Lemma and Lemma 5.3 as follows: Let $\left(h_{n}\right)_{n \in \mathbb{N}}$ be a Dirac sequence such that the functions $\hat{h}_{n}$ have compact supports. For example, $\left(h_{n}\right)_{n \in \mathbb{N}}$ can be defined by (5.1). Since $\widehat{k}(t) \neq 0$ for each $t \in \mathbb{R}$, by Wiener's Division Lemma [23, Lemma 1.4.2] for every $n \in \mathbb{N}$ there exists $q_{n} \in L_{1}(\mathbb{R})$ such that $h_{n}=k * q_{n}$. Now, given $\psi \in C_{b, u}(\mathbb{R})$, we construct a sequence $\left(w_{n}\right)_{n \in \mathbb{N}}$ by the rule $w_{n}=q_{n} * \psi$. Then $w_{n} \in L_{\infty}$ and the sequence $\left(k * w_{n}\right)_{n \in \mathbb{N}}$ takes values in the set $\left\{k * f: f \in L_{\infty}(\mathbb{R})\right\}$. Finally, applying the identities

$$
k * w_{n}=k * q_{n} * \psi=h_{n} * \psi
$$

and Lemma 5.3, we conclude that this sequence converges uniformly to $\psi$. 


\section{Approximation of the eigenvalues' SeQuences by Convolutions}

The idea of this section is to approximate $\gamma_{a}(n)$ by a certain convolution for $n$ large enough. Using the change of variables $r=y^{2}$ in (4.1) we rewrite $\gamma_{a}(n)$ in the form

$$
\gamma_{a}(n)=\int_{\mathbb{R}_{+}} K\left(n, y^{2}\right) 2 y a(y) d y .
$$

By Stirling formula, $K(n, r)$ has the following asymptotic behavior as $n \rightarrow+\infty$ :

$$
K(n, r)=\frac{r^{n} e^{-r}}{n !} \sim \frac{r^{n} e^{n}}{\sqrt{2 \pi} n^{n+1 / 2} e^{r}} .
$$

Using this limit relation and Lebesgue's dominated convergence theorem it is easy to prove that

$$
\lim _{n \rightarrow \infty} \int_{\mathbb{R}_{+}}\left|\frac{r^{n} e^{-r}}{n !}-\frac{r^{n} e^{n}}{\sqrt{2 \pi} n^{n+1 / 2} e^{r}}\right| d r=0 .
$$

We pass from integer $n$ to real $x=\sqrt{n}$ and from $r \geq 0$ to $r=y^{2}$. Consider the function $F$ defined on $[0,+\infty) \times[0,+\infty)$ by

$$
F(x, y)=\frac{y^{2 x^{2}+1} e^{x^{2}}}{x^{2 x^{2}+1} e^{y^{2}}}=\exp \left(\left(2 x^{2}+1\right)(\ln y-\ln x)+x^{2}-y^{2}\right) .
$$

Then (6.2) can be rewritten in the form

$$
\lim _{n \rightarrow \infty} \int_{\mathbb{R}+}\left|K\left(n, y^{2}\right) 2 y-\left(\frac{2}{\pi}\right)^{1 / 2} F(\sqrt{n}, y)\right| d y=0 .
$$

With the change of variables $u=y-x$ we have

$$
\int_{\mathbb{R}_{+}} F(x, y) a(y) d y=\int_{[-x,+\infty)} F(x, x+u) a(x+u) d y,
$$

where

$$
\ln F(x, x+u)=\left(2 x^{2}+1\right) \ln \left(1+\frac{u}{x}\right)-2 x u-u^{2} .
$$

Next, we proceed with some technical lemmas which permit us to analyze the asymptotic behavior of the eigenvalues' sequences at the infinity.

Lemma 6.1 (the integral of the kernel far from the diagonal). For every $\varepsilon>0$ there exists $h>1$ such that the following estimation holds for every $x \geq 1$ :

$$
\int_{[-x,+\infty) \backslash[-h, h]} F(x, x+u) d u \leq \varepsilon .
$$

Proof. Apply the elementary inequality $\ln (1+t) \leq t$ which holds for every $t \geq 0$ :

$$
\ln F(x, x+u) \leq\left(2 x^{2}+1\right) \frac{u}{x}-2 x u-u^{2}=\frac{u}{x}-u^{2} \leq \frac{|u|}{x}-u^{2} .
$$

Suppose that $x \geq 1, h \geq 2$ and $|u| \geq h$. Since $|u| \geq h \geq 2$, we have that $\frac{|u|}{2} \geq 1$. Thus by (6.6) we get

$$
\ln F(x, x+u) \leq \frac{|u|}{x}-u^{2} \leq|u|\left(\frac{|u|}{2}\right)-u^{2}=\frac{u^{2}}{2}-u^{2}=-\frac{u^{2}}{2} .
$$


It follows that

$$
\int_{[-x,+\infty) \backslash[-h, h]} F(x, x+u) d u \leq \int_{[-x,+\infty) \backslash[-h, h]} e^{-\frac{u^{2}}{2}} d u \leq \int_{\mathbb{R} \backslash[-h, h]} e^{-\frac{u^{2}}{2}} d u \leq 2 \int_{h}^{+\infty} e^{-\frac{u^{2}}{2}} d u .
$$

The latter integral tends to zero as $h$ tends to $+\infty$.

Lemma 6.2. Let $L, x \geq 0, h \geq 1$ and $|u| \leq h$. If $h<L<x$, then

$$
\left|F(x, x+u) e^{2 u^{2}}-1\right| \leq e^{5 \frac{h^{3}}{L}}-1 .
$$

Proof. Since $\ln (1+t) \leq t-\frac{t^{2}}{2}+\frac{t^{3}}{3}, \quad t \in(-1,1)$, we obtain for $t=\left|\frac{u}{x}\right| \leq 1$ that

$$
\begin{aligned}
\ln F(x, x+u)+2 u^{2} & =\left(2 x^{2}+1\right) \ln \left(1+\frac{u}{x}\right)-2 x u+u^{2} \\
& \leq\left(2 x^{2}+1\right)\left(\frac{u}{x}-\frac{u^{2}}{2 x^{2}}+\frac{u^{3}}{3 x^{3}}\right)-2 x u+u^{2} \\
& =\frac{u}{x}-\frac{u^{2}}{2 x^{2}}+\frac{u^{3}}{3 x^{3}}+\frac{2 u^{3}}{3 x} \leq \frac{u}{x}+\frac{u^{3}}{x} \leq \frac{5 h^{3}}{L} .
\end{aligned}
$$

On the other hand, by $t-\frac{t^{2}}{2} \leq \ln (1+t)$ for each $t \in[0,1)$, taking $t=\frac{u}{x}$ with $u \in[0, h]$

$$
\begin{aligned}
\ln F(x, x+u)+2 u^{2} & =\left(2 x^{2}+1\right) \ln \left(1+\frac{u}{x}\right)-2 x u+u^{2} \\
& \geq\left(2 x^{2}+1\right)\left(\frac{u}{x}-\frac{u^{2}}{2 x^{2}}\right)-2 x u+u^{2}=\frac{u}{x}-\frac{u^{2}}{2 x^{2}} \geq-\frac{5 h^{3}}{L} .
\end{aligned}
$$

Since $\ln (1-t) \geq-t-\frac{t^{2}}{2}-t^{3}$ for each $t \in[0,2 / 3]$, we take $x>0$ sufficiently large such that $t=-\frac{u}{x} \in[0,2 / 3]$, with $u \in[-h, 0]$. Therefore

$$
\begin{aligned}
\ln F(x, x+u)+2 u^{2} & =\left(2 x^{2}+1\right) \ln \left(1-\left(-\frac{u}{x}\right)\right)-2 x u+u^{2} \\
& \geq\left(2 x^{2}+1\right)\left(\frac{u}{x}-\frac{u^{2}}{2 x^{2}}+\frac{u^{3}}{x^{3}}\right)-2 x u+u^{2} \\
& =\frac{u}{x}-\frac{u^{2}}{2 x^{2}}+\frac{u^{3}}{x^{3}}+\frac{2 u^{3}}{x} \geq-\frac{5 h^{3}}{L} .
\end{aligned}
$$

Combining this calculations we get for all $|u| \leq h$ that

$$
e^{5 \frac{h^{3}}{L}}-1 \geq F(x, x+u) e^{u^{2}}-1 \geq e^{-5 \frac{h^{3}}{L}}-1 \geq-\left(e^{5 \frac{h^{3}}{L}}-1\right) .
$$

Lemma 6.3 ("convoluzation" of the integral operator near the diagonal). Given $\varepsilon>0$ and $h \geq 1$, there exists $L \geq h$ such that for every $x \geq L$

$$
\int_{[-h, h]}\left|F(x, x+u)-e^{-2 u^{2}}\right| d u \leq \varepsilon .
$$

Proof. Suppose that $x \geq L$ and $|u| \leq h$. By Lemma 6.2 for $h \geq 1$ we get

$$
\int_{[-h, h]}\left|F(x, x+u)-e^{-2 u^{2}}\right| d u \leq \int_{[-h, h]} e^{-2 u^{2}}\left|F(x, x+u) e^{2 u^{2}}-1\right| d u \leq 2 h\left(e^{5 h^{3} / L}-1\right) .
$$

The last expression tends to 0 as $L$ tends to $+\infty$. 
Lemma 6.4.

$$
\begin{gathered}
\lim _{x \rightarrow+\infty} \int_{0}^{\infty}\left|F(x, y)-e^{-2(x-y)^{2}}\right| d y=0, \\
\lim _{n \rightarrow \infty} \int_{\mathbb{R}_{+}}\left|K\left(n, y^{2}\right) 2 y-\left(\frac{2}{\pi}\right)^{1 / 2} e^{-2(\sqrt{n}-y)^{2}}\right| d r=0 .
\end{gathered}
$$

Proof. We are going to prove (6.8), then (6.9) will follow by (6.2). Let $\varepsilon>0$. Using Lemma 6.1 choose $h>0$ such that

$$
\int_{[-x,+\infty) \backslash[-h, h]} F(x, x+u) d u \leq \frac{\varepsilon}{3\|a\|_{\infty}}, \quad \int_{[-x,+\infty) \backslash[-h, h]} e^{-2 u^{2}} d u \leq \frac{\varepsilon}{3\|a\|_{\infty}} .
$$

After that using Lemma 6.3 choose $L \geq h$ such that for every $x \geq L$

$$
\int_{[-h, h]}\left|F(x, x+u)-e^{-2 u^{2}}\right| d u \leq \frac{\varepsilon}{3\|a\|_{\infty}} .
$$

Then for every $x \geq L$ the left-hand side of (6.8) is less or equal to $\varepsilon$.

The proofs of this section have many technical details. To be more confident in formula (6.9), we tested it numerically in Wolfram Mathematica. The numerical experiments showed that for every $n \in\{1, \ldots, 1000\}$ the integral in the left-hand side of (6.9) is less than $0.54 / \sqrt{n}$.

Proposition 6.5. Let $a \in L_{\infty}\left(\mathbb{R}_{+}\right)$. Then

$$
\lim _{n \rightarrow+\infty}\left|\gamma_{a}(n)-\left(\frac{2}{\pi}\right)^{1 / 2} \int_{\mathbb{R}_{+}} a(y) e^{-2(y-\sqrt{n})^{2}} d y\right|=0 .
$$

Proof. Write $\gamma_{a}(n)$ as in (6.1), factorize $a(y)$ below the sign of the the integral, estimate $|a(y)|$ by $\|a\|_{\infty}$ and apply (6.9).

There is no surprise that the heat kernel appears in the properties of the eigenvalues' sequences $\gamma_{a}$, because it plays an important role in the theory of Toeplitz operators acting on Fock spaces. In [8] Berger and Coburn characterized some properties of Toeplitz operators $T_{\varphi}$ (boundedness, compactness etc.) by means of its Berezin transform

$$
\widetilde{\varphi}(z)=\frac{1}{\pi} \int_{\mathbb{R}} \varphi(w) e^{-\frac{|z-w|^{2}}{2}} d \nu(w), \quad z \in \mathbb{C},
$$

which is the convolution of the symbol $\varphi$ with the heat kernel $H(w, t)=(4 t \pi)^{-1} e^{-\frac{|w|^{2}}{4 t}}$ at time $t=\frac{1}{2}$. This result holds also for Toeplitz operators with more general symbols (positive Borel measures), see [22].

Our formula (6.10) relates $\gamma_{a}$ with the heat kernel at time $t=\frac{1}{8}$, we denote it simply by $H$ :

$$
H(x)=H(x, 1 / 8)=(2 / \pi)^{1 / 2} e^{-2 x^{2}} .
$$

Lemma 6.6. If $b \in L_{\infty}(\mathbb{R})$ and $a=\chi_{\mathbb{R}_{+}} b$, then

$$
\lim _{x \rightarrow+\infty}|H * a(x)-H * b(x)|=0 .
$$


Proof. The difference in the left-hand side of (6.11) can be estimated as follows:

$$
\begin{aligned}
&|H * a(x)-H * b(x)| \leq\|b\|_{\infty}(2 / \pi)^{1 / 2} \int_{-\infty}^{0} e^{-2(x-y)^{2}} d y \\
& \stackrel{t=x-y}{=}\|b\|_{\infty}(2 / \pi)^{1 / 2} \int_{x}^{+\infty} e^{-2 t^{2}} d t, \quad x \in \mathbb{R}_{+} .
\end{aligned}
$$

Proposition 6.7. Let $\sigma \in \mathrm{RO}\left(\mathbb{Z}_{+}\right)$and $\varepsilon>0$. Then there exist $a \in L_{\infty}\left(\mathbb{R}_{+}\right)$and $N \in \mathbb{N}$ such that

$$
\sup _{n>N}\left|\sigma(n)-\gamma_{a}(n)\right| \leq \varepsilon .
$$

Proof. By Proposition 3.4 there is $f \in \operatorname{RO}([0,+\infty))$ such that $\left.f\right|_{\mathbb{Z}_{+}}=\sigma$ and $\|f\|_{\infty}=$ $\|\sigma\|_{\infty}$. Define $h: \mathbb{R} \rightarrow \mathbb{C}$ as $h(x)=f\left(x^{2}\right)$. Then $h \in C_{b, u}(\mathbb{R})$. Moreover, by Proposition 5.4 there exists $\ell \in L_{\infty}(\mathbb{R})$ such that

$$
\|H * \ell-h\|_{\infty} \leq \frac{\varepsilon}{3}
$$

Denote the restriction $\left.\ell\right|_{\mathbb{R}_{+}}$by $a$. By (6.10) and Lemma 6.6, there are $L_{1}, L_{2}>0$ such that

$$
\left|\gamma_{a}(n)-H * a(\sqrt{n})\right| \leq \frac{\varepsilon}{3}, n \geq L_{1}, \quad|H * \ell(x)-H * a(x)| \leq \frac{\varepsilon}{3}, x \geq L_{2} .
$$

Thus, taking $L=\max \left\{L_{1}, L_{2}\right\}$ by (6.13) and (6.14) one gets for every $n \geq\left\lceil L^{2}\right\rceil$ that

$$
\begin{aligned}
& \left|\gamma_{a}(n)-\sigma(n)\right| \stackrel{x=\sqrt{n}}{\leq}\left|\gamma_{a}\left(x^{2}\right)-H * a(x)\right|+|H * a(x)-H * \ell(x)|+|H * \ell(x)-h(x)| \\
& \leq\left|\gamma_{a}\left(x^{2}\right)-H * a(x)\right|+|H * a(x)-H * \ell(x)|+\|H * \ell-h\|_{\infty} \leq \varepsilon .
\end{aligned}
$$

\section{Density of $\left(\mathfrak{G}\right.$ IN $\operatorname{RO}\left(\mathbb{Z}_{+}\right)$}

In this section we finish the proof of our main result. By Proposition 6.7 we already know that every sequence $\sigma \in \operatorname{RO}\left(\mathbb{Z}_{+}\right)$can be approximated by some eigenvalues' sequences $\gamma_{a}$ for large values of $n$. Thus, it only remains to prove that the sequences vanishing at the infinity can be approximated by eigenvalues' sequences.

Denote by $\mathcal{X}$ the Banach subspace of $L_{\infty}\left(\mathbb{R}_{+}\right)$consisting of all bounded functions $a$ having limit 0 at the infinity.

Lemma 7.1. If $a \in \mathcal{X}$, then $\gamma_{a} \in c_{0}\left(\mathbb{Z}_{+}\right)$.

Proof. Given $\varepsilon>0$, there are $L>0$ and $N_{0} \in \mathbb{Z}_{+}$such that

$$
|a(t)| \leq \frac{\varepsilon}{2}, \quad t \geq L, \quad n^{-1 / 2} \leq \sqrt{\frac{\pi}{2}} \frac{\varepsilon}{\|a\|_{\infty} L^{2}}, \quad n \geq N_{0} .
$$


Thus, by (4.7) and (7.1) we have for every $n \geq N_{0}$ that

$$
\begin{aligned}
\left|\gamma_{a}(n)\right| & \leq \frac{1}{n !}\left[\int_{0}^{L^{2}}|a(\sqrt{r})| e^{-r} r^{n} d r+\int_{L^{2}}^{+\infty}|a(\sqrt{r})| e^{-r} r^{n} d r\right] \\
& \leq \frac{1}{n !}\left[\int_{0}^{L^{2}}|a(\sqrt{r})| e^{-r} r^{n} d r+\frac{\varepsilon}{2} \int_{L^{2}}^{+\infty} e^{-r} r^{n} d r\right] \\
& \leq \frac{1}{n !} \int_{0}^{L^{2}}|a(\sqrt{r})| e^{-r} r^{n} d r+\frac{\varepsilon}{2} \leq \frac{\|a\|_{\infty}}{n !} \int_{0}^{L^{2}} e^{-r} r^{n} d r+\frac{\varepsilon}{2} \\
& \leq \frac{\|a\|_{\infty} e^{-n} n^{n} L^{2}}{n !}+\frac{\varepsilon}{2} \leq \frac{\|a\|_{\infty} L^{2}}{\sqrt{2 n \pi}}+\frac{\varepsilon}{2}=\frac{\varepsilon}{2}+\frac{\varepsilon}{2}=\varepsilon .
\end{aligned}
$$

Theorem 7.2. $\left\{\gamma_{a}: a \in \mathcal{X}\right\}$ is a dense subset of $c_{0}\left(\mathbb{Z}_{+}\right)$.

Proof. The inclusion $\left\{\gamma_{a}: a \in \mathcal{X}\right\} \subseteq c_{0}\left(\mathbb{Z}_{+}\right)$was shown in Lemma 7.1. Unfortunately we were not able to prove the density by constructive tools; the next proof uses nonconstructive duality arguments. By Hahn-Banach theorem, the density of $\left\{\gamma_{a}: a \in \mathcal{X}\right\}$ in $c_{0}\left(\mathbb{Z}_{+}\right)$will be shown if we prove that any continuous linear functional $\varphi$ on $c_{0}\left(\mathbb{Z}_{+}\right)$ that vanishes on $\left\{\gamma_{a}: a \in \mathcal{X}\right\}$ is the zero functional. Thus, let $\phi \in c_{0}\left(\mathbb{Z}_{+}\right)^{*}$ be a linear functional such that $\phi\left(\gamma_{a}\right)=0$ for each $a \in L_{\infty}\left(\mathbb{R}_{+}\right)$. Using the well-known description of the dual space of $c_{0}\left(\mathbb{Z}_{+}\right)$we find a sequence $p=\left(p_{n}\right)_{n \in \mathbb{Z}_{+}} \in \ell_{1}\left(\mathbb{Z}_{+}\right)$such that

$$
\phi(y)=\sum_{n=0}^{\infty} p_{n} y_{n} \quad y \in c_{0}\left(\mathbb{Z}_{+}\right) .
$$

Then we have that

$$
0=\phi\left(\gamma_{a}\right)=\sum_{n=0}^{\infty} \gamma_{a}(n) p_{n}, \quad a \in L_{\infty}\left(\mathbb{R}_{+}\right) .
$$

In particular, substituting $a=\chi_{[0, x]} \in \mathcal{X}$ with $0 \leq x<+\infty$, we obtain

$$
0=\sum_{n=0}^{\infty} \gamma_{a}(n) p_{n}=\int_{0}^{x} \sum_{n=0}^{\infty} p_{n} K(n, r) d r .
$$

The function $r \mapsto \sum_{n=0}^{\infty} p_{n} K(n, r)$, being the sum of a uniformly converging series of continuous functions, is continuous, and by the first fundamental theorem of calculus, $\sum_{n=0}^{\infty} p_{n} K(n, r)=0, r \geq 0$. Now, replace $K(n, r)$ by $r^{n} e^{-r} / n$ ! and factorize $e^{-r}$ :

$$
\sum_{n=0}^{\infty} \frac{p_{n} r^{n}}{n !}=0 \quad r \geq 0 .
$$

Denote by $f$ the function

$$
f(z)=\sum_{n=0}^{\infty} \frac{p_{n} z^{n}}{n !} .
$$

Since $p_{n} \rightarrow 0$, the root test shows that $f$ is an entire function. The equality (7.2) says that $f(r)=0$ for every $r \geq 0$. Therefore $f$ is the zero constant, and all coefficients $p_{n}$ are zero.

Now we are ready to prove the main result of the paper. 
Theorem 7.3. 05 is dense in $\mathrm{RO}\left(\mathbb{Z}_{+}\right)$.

Proof. Let $\sigma \in \operatorname{RO}\left(\mathbb{Z}_{+}\right)$and $\varepsilon>0$. By Proposition 6.7 there are $b \in L_{\infty}\left(\mathbb{R}_{+}\right)$and $N \in \mathbb{Z}_{+}$such that

$$
\left|\sigma(n)-\gamma_{b}(n)\right| \leq \frac{\varepsilon}{2}, \quad n>N
$$

Define $\vartheta=(\vartheta(n))_{n \in \mathbb{Z}_{+}}$by

$$
\vartheta(n)= \begin{cases}\sigma(n)-\gamma_{b}(n), & \text { if } n \leq N, \\ 0 & \text { otherwise. }\end{cases}
$$

Thus $\vartheta \in c_{0}\left(\mathbb{Z}_{+}\right)$, and by Theorem 7.2 there exists $c \in L_{\infty}\left(\mathbb{R}_{+}\right)$such that

$$
\left\|\vartheta-\gamma_{c}\right\|_{\infty} \leq \frac{\varepsilon}{2}
$$

Taking $a=b+c \in L_{\infty}\left(\mathbb{R}_{+}\right)$one gets that

$$
\left\|\sigma-\gamma_{a}\right\|_{\infty} \leq\left\|\sigma-\gamma_{b}-\vartheta\right\|_{\infty}+\left\|\vartheta-\gamma_{c}\right\|_{\infty} \leq \sup _{n>N}\left|\sigma(n)-\gamma_{b}(n)\right|+\frac{\varepsilon}{2} \leq \varepsilon .
$$

\section{BEYOND THE CLASS OF BOUNDED GENERATING SYMBOLS}

In this section we indicate a class of functions wider than $L_{\infty}(\mathbb{R})$, with eigenvalues' sequences belonging to $\operatorname{RO}\left(\mathbb{Z}_{+}\right)$. Furthermore, we give an unbounded generating symbol $a$ such that $\gamma_{a} \in \ell_{\infty}\left(\mathbb{Z}_{+}\right) \backslash \operatorname{RO}\left(\mathbb{Z}_{+}\right)$.

Following [18] we denote by $L_{1}^{\infty}\left(\mathbb{R}_{+}, e^{-r^{2}}\right)$ the subspace of all measurable functions $a$ on $\mathbb{R}_{+}$for which the following integrals are finite for all $n \in \mathbb{Z}_{+}$:

$$
\int_{\mathbb{R}_{+}}|a(r)| e^{-r^{2}} r^{n} d r<+\infty
$$

For $a \in L_{1}^{\infty}\left(\mathbb{R}_{+}, e^{-r^{2}}\right)$, we consider the following averages [18]:

$$
\mathcal{B}_{(j)} a(r)=\int_{r}^{+\infty} \mathcal{B}_{(j-1)} a(u) e^{r-u} d u, \quad j=1,2, \ldots,
$$

where $\mathcal{B}_{(0)} a(r)=a(\sqrt{r})$. Integrating by parts $j$ times one can express $\gamma_{a}$ through $\mathcal{B}_{(j)} a$ :

$$
\gamma_{a}(n)=\frac{1}{(n-j) !} \int_{\mathbb{R}_{+}} \mathcal{B}_{(j)} a(r) r^{n-j} e^{-r} d r=\gamma_{\mathcal{B}_{(j) a} \circ \mathrm{sq}}(n-j), \quad n \geq j,
$$

where $\operatorname{sq}(x)=x^{2}, x \in \mathbb{R}_{+}$. It is easily seen that if $\mathcal{B}_{(j)} a \in L_{\infty}\left(\mathbb{R}_{+}\right)$for some $j \in \mathbb{Z}_{+}$, then the eigenvalues' sequence (and the corresponding Toeplitz operator) is bounded. The definition of the averages $\mathcal{B}_{(j)} a$ and the facts mentioned above are taken from [18, Section 4].

Let us denote by $\mathcal{M}$ the class of symbols $a \in L_{1}^{\infty}\left(\mathbb{R}_{+}, e^{-r^{2}}\right)$ such that the average (8.2) is bounded for some $j \in \mathbb{Z}_{+}$:

$$
\mathcal{M}:=\left\{a \in L_{1}^{\infty}\left(\mathbb{R}_{+}, e^{-r^{2}}\right): \mathcal{B}_{(j)} a \in L_{\infty}\left(\mathbb{R}_{+}\right) \text {for some } j \in \mathbb{Z}_{+}\right\} .
$$

Proposition 8.1. If $a \in \mathcal{M}$, then $\gamma_{a} \in \mathrm{RO}\left(\mathbb{Z}_{+}\right)$. 
Proof. Let $j \in \mathbb{Z}_{+}$and $a \in L_{1}^{\infty}\left(\mathbb{R}_{+}, e^{-r^{2}}\right)$ such that $\mathcal{B}_{(j)} a \in L_{\infty}\left(\mathbb{R}_{+}\right)$. Since $\gamma_{a}(n)=$ $\gamma_{\mathcal{B}_{(j) a} \circ \mathrm{sq}}(n-j)$, with $\operatorname{sq}(x)=x^{2}, x \in \mathbb{R}_{+}$, one gets by (4.2) and (4.5) that for every $n>j$

$$
\begin{aligned}
\sqrt{n+1}\left|\gamma_{a}(n)-\gamma_{a}(n+1)\right| & =\sqrt{n+1}\left|\gamma_{\mathcal{B}_{(j)} a \circ \mathrm{sq}}(n-j+1)-\gamma_{\mathcal{B}_{(j)} a \circ \mathrm{sq}}(n-j)\right| \\
& \leq\left\|\mathcal{B}_{(j)} a \circ \mathrm{sq}\right\|_{\infty} \kappa(n-j, n-j+1) \sqrt{n+1} \\
& \leq\left\|\mathcal{B}_{(j)} a \circ \mathrm{sq}\right\|_{\infty} \sqrt{\frac{2(n+1)}{\pi(n-j+1)}} .
\end{aligned}
$$

Hence $\sup _{n \in \mathbb{Z}_{+}}\left(\sqrt{n+1}\left|\gamma_{a}(n)-\gamma_{a}(n+1)\right|\right)<\infty$ and, by Proposition 3.2, the eigenvalue sequence $\gamma_{a}$ is Lipschitz continuous with respect $\rho$.

Folland [14, Lemma 2.95] proved that for the class of unbounded measurable symbols $a \in L_{1}^{\infty}\left(\mathbb{R}_{+}, e^{-r^{2}}\right)$ which satisfy the inequality

$$
|a(r)| \leq \text { const } e^{\delta r^{2}}, \quad \text { for some } \delta<1,
$$

the linear mapping $a \mapsto T_{a}$ is injective. However, this class contains defining symbols which generate eigenvalues' sequences do not belonging to $\operatorname{RO}\left(\mathbb{Z}_{+}\right)$.

Example 8.2. Let $\delta=1-\frac{1}{\sqrt{2}}$. Then the function $a$ defined by the rule

$$
a(r)=e^{\left(\delta-\frac{i}{\sqrt{2}}\right) r^{2}}
$$

satisfies (8.5) and belongs to $L_{1}^{\infty}\left(\mathbb{R}_{+}, e^{-r^{2}}\right)$. Let us calculate the corresponding eigenvalue's sequence using the change of variables $t=\sqrt{2} r$ and the formula [15, Eq. 3.381-5]:

$$
\gamma_{a}(n)=\frac{1}{n !} \int_{\mathbb{R}_{+}} e^{\left(1-\frac{1}{\sqrt{2}}-\frac{i}{\sqrt{2}}\right) r} e^{-r} r^{n} d r=\frac{2^{\frac{n+1}{2}}}{n !} \int_{\mathbb{R}_{+}} e^{-(1+i) t} t^{n} d t=e^{-i(n+1) \frac{\pi}{4}} .
$$

The sequence of its consecutive differences is given by

$$
\gamma_{a}(n+1)-\gamma_{a}(n)=e^{-i(n+2) \frac{\pi}{4}}\left(1-e^{i \frac{\pi}{4}}\right)
$$

and does not converge to 0 , though $\rho(n+1, n) \rightarrow 0$. Thus $\gamma_{a} \in \ell_{\infty}\left(\mathbb{Z}_{+}\right) \backslash \operatorname{RO}\left(\mathbb{Z}_{+}\right)$.

Acknowledgments. The authors are grateful to Professor Nikolai Vasilevski for introducing to us the world of commutative $\mathrm{C}^{*}$-algebras of Toeplitz operators. Many ideas used in the proofs come from our joint papers with Crispin Herrera Yañez, Ondrej Hutník, and Nikolai Vasilevski. The second author was partially supported by the project IPN-SIP 2015-0422.

\section{REFERENCES}

[1] L. D. Abreu, N. Faustino, On Toeplitz operators and localization operators. Proc. Am. Math. Soc. (to appear).

[2] V. Bargmann, On a Hilbert space of analytic functions and an associated integral transform. Comm. Pure Appl. Math. 3 (1961), 187-214. http: //dx . doi.org/10.1002/cpa.3160140303

[3] W. Bauer, C. Herrera Yañez, and N. Vasilevski, Eigenvalue characterization of radial operators on weighted Bergman spaces over the unit ball. Integr. Equ. Oper. Theory 782 (2014), 271-300. http: //dx . doi .org/10.1007/s00020-013-2101-1 
[4] W. Bauer, T. Issa, Commuting Toeplitz operators with quasi-homogeneous symbols on the SegalBargmann space. J. Math. Anal. Appl. 386 (2012), 213-235.

http://dx. doi.org/10.1016/j.jmaa.2011.07.058

[5] W. Bauer, H. Le, Algebraic properties and the finite rank problem for Toeplitz operators on the Segal-Bargmann space. J. Funct. Anal. 261 (2011), 2617-2640.

http://dx.doi.org/10.1016/j.jfa.2011.07.006

[6] F. A. Berezin, General concept of quantization. Comm. Math. Phys. 40 (1975), 153-174. http://dx.doi.org/10.1007/BF01609397

[7] C. A. Berger, L. A. Coburn, Toeplitz operators and quantum mechanics. J. Funct. Anal. 68 (1986), 273-299. http://dx.doi.org/10.1016/0022-1236(86)90099-6

[8] C. A. Berger, L. A. Coburn, Toeplitz operators on the Segal-Bargmann space. Trans. Amer. Math. Soc. 2 (1987), 813-829. http://dx. doi .org/10.2307/2000671

[9] L. A. Coburn, Deformation estimates for the Berezin-Toeplitz quantization. Commun. Math. Phys. 149 (1992), 415-424. http://projecteuclid.org/euclid.cmp/1104251229

[10] M. Engliš, Toeplitz operators and group representations. J. Fourier Anal. Appl. 13 (2007), 243265.

http://dx.doi.org/10.1007/s00041-006-6009-x

[11] K. Esmeral, E. A. Maximenko, $C^{*}$-algebra of angular Toeplitz operators on Bergman spaces over the upper half-plane. Commun. Math. Anal. 17 (2014), no. 2, 151-162. http://projecteuclid.org/euclid.cma/1418919761

[12] V. A. Fock, Konfigurationsraum und zweite Quantelung. Z. Phys. 75 (1932), 622-647. http://dx.doi.org/10.1007/BFQ1344458

[13] G. B. Folland, A course in Abstract Harmonic Analysis. Studies in Advanced Mathematics, 1995.

[14] G. B. Folland, Harmonic Analysis in Phase Space. Princeton University Press, 1989.

[15] I. S. Gradshteyn, I. M. Ryzhik, Table of Integrals, Series and Product. Academic Press, 1980.

[16] S. Grudsky, A. Karapetyants, and N. Vasilevski, Dynamics of properties of Toeplitz operators with radial symbols. Integr. Equ. Oper. Theory 20 (2004), 217-253. http://dx. doi.org/10.1007/s00020-003-1295-z

[17] S. M. Grudsky, E. A. Maximenko, and N. L. Vasilevski, Radial Toeplitz operators on the unit ball and slowly oscillating sequences. Commun. Math. Anal. 14 (2013), no. 2, 77-94. http: //projecteuclid.org/euclid.cma/1356039033

[18] S. Grudsky, N. Vasilevski, Toeplitz operators on the Fock space: Radial component effects. Integr. Equ. Oper. Theory 44 (2002), 10-37. http://dx.doi.org/10.1007/BF01197858

[19] C. Herrera Yañez, O. Hutník, and E. A. Maximenko, Vertical symbols, Toeplitz operators on weighted Bergman spaces over the upper half-plane and very slowly oscillating functions. C. R. Acad. Sci. Paris, Ser. I 352 (2014), 129-132.

http://dx. doi.org/10.1016/j . crma.2013.12.004

[20] C. Herrera Yañez, E. A. Maximenko, N. L. Vasilevski, Radial Toeplitz operators revisited: Discretization of the vertical case. Accepted in Integral Equations and Operator Theory. http: //dx.doi .org/10.1007/s00020-014-2213-2

[21] E. Hewitt, K. A. Ross, Abstract Harmonic Analysis II. A Serie of Comprehensive Studies in Mathematics, Springer-Verlag, 1970.

[22] J. Isralowitz, K. Zhu, Toeplitz operators on Fock spaces. Integr. Equ. Oper. Theory 66 (2010), 593-611. http://dx.doi.org/10.1007/s00020-010-1768-9

[23] H. Reiter, J. D. Stegeman, Classical Harmonic Analysis and Locally Compact Groups. 2nd Edition, Oxford University Press, 2001.

[24] I. E. Segal, Lectures at the summer seminar on Applied Math. Boulder, Colorado, 1960. 
[25] D. Suárez, The eigenvalues of limits of radial Toeplitz operators. Bull. London Math. Soc. 40 (2008), 631-641.

http://dx.doi.org/10.1112/blms/bdn042

[26] N. L. Vasilevski, Commutative Algebras of Toeplitz Operators on the Bergman Space. Operator Theory: Advances and Applications, vol. 185, Birkhäuser, 2008.

[27] N. Zorboska, The Berezin transform and radial operators. Proc. Amer. Math. Soc. 131 (2003), 793-800.

http: //www . jstor . org/stable/1194482

[28] K. Zhu, Analysis on Fock Spaces. In series: Graduate Texts in Mathematics, vol. 263, Springer, 2012.

${ }^{1}$ Department of Mathematics, CinVESTAV-IPN, México, D.F, 07360, Mexico

E-mail address: matematikoua@gmail.com

${ }^{2}$ Escuela Superior de Física y Matemáticas, Instituto Politécnico Nacional, México, D.F, 07730, Mexico

E-mail address: maximenko@esfm.ipn.mx 